sites. Signal 2 often comes from cytokine proteins and cell-surface growth factors made by a second class of immune cell, helper T cells, that recognize the same antigen. In other words, the production of circulating antibodies in response to many pathogens depends on recognition of those pathogens by both T and B cells. This constraint greatly reduces the risk of autoantibody production, as $\mathrm{T}$ and $\mathrm{B}$ cells would have to escape their tolerance to the same self-antigen simultaneously.

For several important classes of microorganism, however, signal 2 can come from specific components of the pathogens themselves, rather than through $\mathrm{T}$ cells. The best understood of these T-cell-independent antigens is lipopolysaccharide, a major constituent of the cell wall of Gramnegative bacteria. The unique lipid group of lipopolysaccharide is a structural motif found in most such bacteria, and is recognized by a surface receptor present on all B cells, Toll-like receptor 4 (TLR4) ${ }^{3}$. TLR4 signals through an ancient pathogen-sensing pathway that was first discovered in fruit-flies and involves the Myd88 protein and the gene-transcription factor NF- $\mathrm{KB}$. High concentrations of lipopolysaccharide stimulate the proliferation of all B cells. But at lower concentrations, the simultaneous recognition of lipopolysaccharide by antigen receptors and by TLR4 causes a synergistic signal, which provokes proliferation and production of circulating antibodies selectively by bacteria-specific B cells

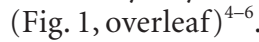

Leadbetter et al. ${ }^{1}$ now reveal that, in a mouse model of systemic autoimmune diseases, the simultaneous activation of cell-surface antigen receptors and another Toll-like receptor, TLR9, causes a particular subclass of self-immunoglobulin - IgG2a, part of the IgG class - to be recognized by $\mathrm{B}$ cells as if it were a pathogen. This triggers T-cell-independent proliferation of the B cells.

The authors took advantage of a genetically engineered mouse strain in which most B cells bear surface antibodies with low affinity for self-IgG2a. Previous experiments showed that, because this affinity is low, binding of self-IgG2a is not normally enough to trigger signal 1 , so the immunoglobulin neither activates the B cells nor makes them tolerant. But when the mice are bred with a strain that is prone to several autoimmune disorders (systemic lupus erythematosus, vasculitis and arthritis), the self-IgG2a in the offspring becomes potently 'immunogenic', resulting in high concentrations of circulating rheumatoid-factor autoantibodies ${ }^{7}$.

It was not clear why this should happen. But Leadbetter et al. ${ }^{1}$ find that, in the bloodstream of the autoimmune mice, self-IgG2a accumulates in complexes with self-DNA.

Astronomy

\title{
The wanderer returns
}

Astronomers are celebrating the return of a comet last seen during the seventeenth century. Ikeya-Zhang, named after the Japanese and Chinese amateur astronomers who spotted it on 1 February of this year, is the brightest comet seen from Earth since Hale-Bopp's visit in 1997. Although comets with longer periods may exist, for the time being Ikeya-Zhang has set a new record: its return after

341 years gives it the longest period of any comet so far observed making successive orbits of the Sun.

The historical records of periodicity cannot be interpreted with certainty but they are persuasive. After observing the comet for about 20 days, Brian Marsden of the Smithsonian Astrophysical Observatory in Cambridge, Massachusetts, calculated that the period of the comet is 341 years. This, and the shape of its trajectory, match it to a comet first seen by Polish astronomer Johannes Hevelius in 1661. Hevelius's comet was one of those later used by Edmond Halley — of Halley's comet fame - in his treatise on comet periodicity. Chinese astronomical records also report a comet in the sky in

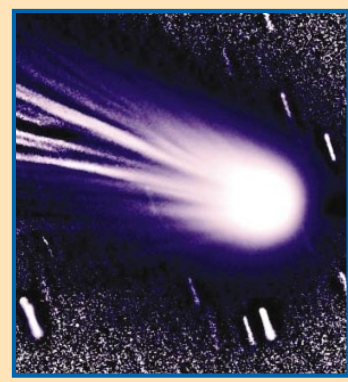

the years 1320 and 979 , which would tally with Ikeya-Zhang's 341-year period, but these sightings may not be reliable.

Comets are essentially dirty snowballs of dust and ice, and are thought to be the remnants of the galactic debris that formed the planets of our Solar System. Measurements of the composition and temperature of the dust and ionized gas trailing behind Ikeya-Zhang are in progress, and will be of particular interest to astronomers. Because of its lengthy periodicity, Ikeya-Zhang makes far fewer trips to the warmer climes closer to the Sun than shorterperiod comets such as Halley's (which returns at 76-year intervals), and may have quite a different make-up

as a result.

The comet is now around
70 million kilometres from Earth and at its closest approach, on 29 April, it will pass within 60 million kilometres. Since it was spotted a couple of months ago, Ikeya-Zhang has put on quite a display for stargazers. Initially, its tail appeared long and wispy, but now it is short and stubby, so that the comet resembles a shuttlecock. Last week, it seemed to graze the bright 'star' of the giant spiral galaxy, Andromeda. But it wasn't such a close shave Andromeda is 2.5 million light years away.

Seen from Earth, Ikeya-Zhang is moving over the North Pole and should be visible as a faint smudge to observers in the Northern Hemisphere throughout the night. The best time for viewing is before sunrise, when the comet is about 15 degrees above the horizon (the width of a hand held at arm's length roughly marks out 15 degrees). Although it is visible to the naked eye, its brightness varies, and a pair of binoculars or a small telescope will enhance the show. After 29 April, it will gradually fade from view - and won't return until 2343. Tom Clarke
The DNA is presumably released during normal or pathological cell death, and (for unknown reasons) fails to be cleared from the bloodstream. TLR9 usually serves as a pathogen sensor by detecting certain motifs (unmethylated $\mathrm{CpG}$ base pairs) that are more common in bacterial than mammalian DNA ${ }^{8}$. But these features are also found in certain parts of mammalian genes, and Leadbetter et al. show that the ability of self-IgG2a to stimulate an immune response results from its recognition by cell-surface antigen receptors and the simultaneous detection of associated selfDNA by TLR9 on B cells (Fig. 1). When the association is broken, or the TLR9-triggered cellular signalling pathway is disrupted, IgG2a loses this ability.

High levels of rheumatoid factors against IgG are not only found in autoimmune-prone mice. They also occur in people with diseases such as rheumatoid arthritis and Sjögren's syndrome - in which the immune system attacks the joints and, in Sjögren's syndrome, the tissues that produce tears and saliva - as well as in some patients with lupus erythematosus, which affects many more tissues. Although we do not know how rheumatoid factors are involved in these diseases, a high titre at diagnosis correlates with the severity both of later rheumatoid arthritis and of disease in tissues other than joints, such as blood vessels. Leadbetter et al.'s results ${ }^{1}$ offer an explanation for how the rheumatoid factors are produced. And they may explain another puzzling observation - that rheumatoid-factor autoantibodies are also generated during normal immune responses to acute bacterial, viral and mycobacterial infections. Foreign DNA, like self-DNA, might form macromolecular complexes with IgG, synergistically triggering IgG-binding antigen receptors and 